\title{
GCU
}

Glasgow Caledonian

University

University for the Common Good

\section{Partial discharge signal denoising based on VMD and group-sparse TV denoising}

Dhandapani, Ragavesh ; Mitiche, Imene; Sarma Mallela, Venkateswara ; Morison, Gordon

Published in:

2019 2nd International Conference on Signal Processing and Information Security (ICSPIS)

DOI:

10.1109/ICSPIS48135.2019.9045901

Publication date:

2020

Document Version

Author accepted manuscript

Link to publication in ResearchOnline

Citation for published version (Harvard):

Dhandapani, R, Mitiche, I, Sarma Mallela, V \& Morison, G 2020, Partial discharge signal denoising based on VMD and group-sparse TV denoising. in 2019 2nd International Conference on Signal Processing and Information Security (ICSPIS). IEEE, 2nd International Conference on Signal Processing and Information Security , Dubai, United Arab Emirates, 30/10/19. https://doi.org/10.1109/ICSPIS48135.2019.9045901

\section{General rights}

Copyright and moral rights for the publications made accessible in the public portal are retained by the authors and/or other copyright owners and it is a condition of accessing publications that users recognise and abide by the legal requirements associated with these rights.

Take down policy

If you believe that this document breaches copyright please view our takedown policy at https://edshare.gcu.ac.uk/id/eprint/5179 for details of how to contact us. 


\title{
Partial Discharge Signal Denoising based on VMD and Group-Sparse TV Denoising
}

\author{
Ragavesh Dhandapani*, Imene Mitiche ${ }^{\dagger}$, Venkatewara Sharma Mallela* and Gordon Morison ${ }^{\dagger}$ \\ * College of Engineering \\ National University of Science and Technology \\ PB 2322, CPO Seeb 111, Sultanate of Oman. \\ E-mail: ragavesh@nu.edu.om \\ $\dagger$ Department of Computing \\ Glasgow Caledonian University \\ 70 Cowcaddens Road, Glasgow G40BA, UK.
}

\begin{abstract}
The partial discharge signal measurement is a non-destructive diagnostics procedure to assess the status of insulation system in high voltage equipment. Due to the adverse measurement conditions, the PD signals are strongly influenced with external noise. In this paper, a new approach is presented for PD signal denoising, which combines the Variational Mode Decomposition and Group-Sparse Total Variation based denoising. The proposed method is applied to extract the simulated PD signal buried in white noise, discrete spectral interference and color noise. Simulation results show that the performance of the proposed VMD-GSTV method is superior to that of Wavelet and the recently introduced Wavelet Total Variation method, specifically when the SNR is low.
\end{abstract}

Index Terms-Denoising; Group-Sparse Total Variation; Partial Discharge; Variational Mode Decomposition

\section{INTRODUCTION AND RELATED WORK}

High Voltage (HV) equipment failure occurs as a result of insulation deterioration initiated by voids created in the insulation material from the combined effects of thermal, electrical, mechanical, and environmental stresses during long-term operation. Due to the high voltage stress, localized dielectric breakdown of a small portion of an insulator occurs, resulting in a Partial Discharge (PD) signal. Over the time, repetitive discharge causes permanent breakdown of insulating materials which leads to complete breakdown of the equipment[1].

The most common on-site noise reported during PD measurements are noise from corona discharge, commutator noise, white Gaussian noise, thermal noise, pink noise, and highfrequency signal interference from communication equipment, commonly referred as Discrete Spectral Interference (DSI) [24]. For the past few years, many researchers proposed signal decomposition based denoising algorithms for PD signals, such as Wavelet Transform (WT) [2-5], Empirical Mode Decomposition (EMD) [6, 7], Variational Mode Decomposition (VMD) [8]. Wavelet transform is considered to be one of the popular method, WT using Discrete Wavelet Transform (DWT) with hard and soft thresholding for denoising PD signal is applied in [2-4] and Adaptive Dual Tree Complex Wavelet Transform (ADTCWT) with automatic threshold by applying adaptive singular value decomposition [5]. However, the performance of Wavelet denoising relied on the type, order, level of decomposition and threshold type - not practical for certain applications.

Apart from WT, adaptive signal decomposition techniques were used in many denoising applications [9]. EMD is an adaptive signal decomposition technique, which decomposes a signal into series of Intrinsic Mode Functions (IMFs) with the different frequency levels. EMD with Interval Thresholding (IT), Iterative IT (IIT) and Clear IIT (CIIT) were used in PD denoising [6]. Recently, a Novel Adaptive Ensemble EMD (NAEEMD) was applied to denoising PD signal [7], however, in all these EMD-based methods the IMFs are taken as mean of many trials, which results in a large amount of computation time and other issues such as error accumulation, mode mixing and end effects [10].

To overcome these issues, Dragomiretskiy and Zosso [11] proposed VMD, a non-recursive approach with a firm mathematical foundation. It decomposes a multi-component input signal into a set of Band Limited IMFs (BLIMFs). Hybrid of VMD and Wavelet Packet Transform (WPT) were applied to synthetic and real-time PD denoising and VMD based WT is applied in denoising UHF PD signals [8]. In spite of the noise sources considered in the literature i.e. white noise and narrow band noise, the performance of VMD need to be studied for other various noise sources.

Total Variation Denoising (TVD) is an optimization problem for removal of noise in 2-dimensional data i.e. image [12]. Selesnick and Condat [13, 14] developed 1-dimensional TVD algorithm, applied to an electrocardiogram (ECG) signal and vibration signal denoising [15]. Signals processed using TVD often exhibit stair-case artifacts [16], Selesnick and Chen proposed Group-Sparse Total Variation (GSTV) denoising which is suitable when the signal to be estimated is known to be group sparse, which is more suitable for PD signal denoising. It is an iterative algorithm derived using the MajorizationMinimization (MM) optimization method with fast solvers for banded systems of linear equations.

Usage of various decomposition methods and application of total variation denoising in PD signal is still an ongoing area of research. A new denoising approach based on VMD and GSTV techniques for PD signal corrupted with white noise, color noise and DSI is proposed in this paper. 


\section{Methodology}

The electrical detection method uses matching impedance as either RC or RLC circuit for wide and narrow band PD detection [2]. The output pulse shape is based on the type of detection circuit, which is realized as natural response of either parallel RC or RLC circuit, they are Damped Exponential Pulse (DEP) and Damped Oscillatory Pulse (DOP). In this work, DOP model along with white noise, DSI and color noise is considered for implementation. The PD signal $P D_{D O P}(t)$ is modeled using Equation (1) as given below,

$$
P D_{D O P}(t)=A\left(e^{-\alpha t}-e^{-\beta t}\right) \sin (\omega t)
$$

where $A$ represents the amplitude of the PD signal, $\alpha$ and $\beta$ are the damping factors and $\omega$ is the damping frequency. The PD signal with the random amplitude between $0.7 \mathrm{~V}$ to $1.3 \mathrm{~V}$, $\alpha$ is set as $7.5 \times 10^{5}, \beta$ is set as $16 \times 10^{6}$ and $\omega$ as $150 \mathrm{kHz}$ is considered. The sampling frequency $f_{s}$ is set to $20 \mathrm{MHz}$.

First noise model is a commonly-used random noise process is white noise. The built-in additive white Gaussian noise model from MATLAB ${ }^{\circledR}$ used with Signal-to-Noise Ratio (SNR) of $-16 \mathrm{~dB},-12 \mathrm{~dB},-8 \mathrm{~dB}$ and $-4 \mathrm{~dB}$. The second noise model is the DSI [4], the presence of continuous sinusoidal noise from the communication systems represented in the form of combination of amplitude modulated signals given by the Equation (2).

$$
n_{A M}(t)=\sum_{c=1}^{9}\left(A_{c}+m * \sin \left(\omega_{m} t\right)\right) * \sin \left(\omega_{c} t\right)
$$

the carrier amplitude $A_{c}=1$, frequency of the carrier signal $\omega_{c}=0.1-1.7 \mathrm{MHz}$ in steps of $200 \mathrm{kHz}$, the modulation depth $m=0.4$ and the frequency of the modulating wave $\omega_{m}=$ $1 k H z$. A third noise model is additive pink noise simulated using MATLAB ${ }^{\circledR}$ function ColoredNoise.

The thermal noise generated by the detection system and the background noise are considered as white noise and the interference from radio transmission is considered as DSI [3, 4] and pink noise ( $\frac{1}{f}$ noise) [17]. A typical on-site PD signals were simulated using Equation (1) added with the different white noise levels and the presence/absence of DSI and color noise as shown in Table I. These twelve noisy PD signals are subjected to the proposed VMD-GSTV algorithm and the obtained results are compared with wavelet denoising methods. The following section describes the proposed VMD-GSTV.

\section{MAthematicAl BACKGROUND}

\section{A. Variational Mode Decomposition (VMD)}

VMD can non-recursively decomposes a real valued input signal $f$ into a set of $K$ sub-signals (modes), $u_{k}$, that have specific sparsity. Each mode $k$ is compact around a central

TABLE I: Simulated Noisy PD Signal

\begin{tabular}{|l|c|c|c|}
\hline PD Signal & \multicolumn{3}{|c|}{ DOP using Equation (1) } \\
\hline Noise Type & AWGN & AWGN+DSI & AWGN+DSI+Color \\
\hline AWGN Levels & \multicolumn{3}{|c|}{$-16 \mathrm{~dB},-12 \mathrm{~dB},-8 \mathrm{~dB},-4 \mathrm{~dB}$} \\
\hline Noisy PD signals & $S_{1}$ to $S_{4}$ & $S_{5}$ to $S_{8}$ & $S_{9}$ to $S_{12}$ \\
\hline
\end{tabular}

frequency $\omega_{k}$, and the input signal is the sum of these components [11].

The process of signal decomposition is to solve a constrained variational problem is written as:

$$
\begin{gathered}
\min _{\left\{u_{k}\right\},\left\{\omega_{k}\right\}}\left\{\sum_{k=1}^{K}\left\|\frac{\partial}{\partial_{t}}\left[\left(\delta(t)+\frac{j}{\pi t}\right) * u_{k}(t)\right] e^{-j \omega_{k} t}\right\|_{2}^{2}\right\} \\
\text { subject to } \sum_{k=1}^{K} u_{k}=f(t)
\end{gathered}
$$

where $\left\{u_{k}\right\}=\left\{u_{1}, \ldots, u_{K}\right\}$ and $\left\{\omega_{k}\right\}=\left\{\omega_{1}, \ldots, \omega_{K}\right\}$ are the mode components and their center frequencies, $K$ is the number of modes to be recovered, $\delta(t)$ denotes impulse function and $f$ is the input signal.

To solve the Equation (3), constrained variational problem is transformed into unconstrained. This is achieved by introducing the Lagrangian multiplier $(\lambda)$ and quadratic penalty term $\alpha$. The new unconstrained problem is as follows:

$$
\begin{gathered}
\mathcal{L}\left(\left\{u_{k}\right\},\left\{\omega_{k}\right\}, \lambda\right)= \\
\alpha \sum_{k=1}^{K}\left\|\frac{\partial}{\partial_{t}}\left[\left(\delta(t)+\frac{j}{\pi t}\right) * u_{k}(t)\right] e^{-j \omega_{k} t}\right\|_{2}^{2} \\
+\left\|f(t)-\sum_{k=1}^{K} u_{k}(t)\right\|_{2}^{2}+\left\langle\lambda(t), f(t)-\sum_{k=1}^{K} u_{k}(t)\right\rangle
\end{gathered}
$$

The solution of Equation (4) now can be found as the saddle point of the augmented Lagrangian in a sequence of iterative sub-optimizations referred as Alternate Direction Method of Multipliers (ADMM)[11]. The optimization procedure of VMD includes the following steps:

1) Initialize modes $\left\{\hat{u}_{k}^{1}\right\}$, center frequency $\left\{\omega_{k}^{1}\right\}$, and $\hat{\lambda}^{1}$. Set $n=0$

2) Update the modes $\hat{u}_{k}$ for all $\omega \geq 0$ :

$$
\hat{u}_{k}^{n+1}(\omega)=\frac{\hat{f}(\omega)-\sum_{i<k} \hat{u}_{i}^{n+1}(\omega)-\sum_{i>k} \hat{u}_{i}^{n}(\omega)+\frac{\hat{\lambda}^{n}(\omega)}{2}}{1+2 \alpha\left(\omega-\omega_{k}^{n}\right)^{2}}
$$

3) Update the center frequencies $\omega_{k}$ :

$$
\omega_{k}^{n+1}=\frac{\int_{0}^{\infty} \omega\left|\hat{u}_{k}^{n+1}(\omega)\right|^{2} d \omega}{\int_{0}^{\infty}\left|\hat{u}_{k}^{n+1}(\omega)\right|^{2} d \omega}
$$

4) Update dual ascent for all $\omega \geq 0$ :

$$
\hat{\lambda}^{n+1}(\omega) \leftarrow \hat{\lambda}^{n}(\omega)+\tau\left(\hat{f}(\omega)-\sum_{k} \hat{u}_{k}^{n+1}(\omega)\right)
$$

5) Repeat step 2 - 4, until convergence:

$$
\frac{\sum_{k}\left\|\hat{u}_{k}^{n+1}-\hat{u}_{k}^{n}\right\|_{2}^{2}}{\left\|\hat{u}_{k}^{n}\right\|_{2}^{2}}<\epsilon
$$

The noisy PD signal is decomposed into 5 modes $(\mathrm{K}=5)$ and their spectrum are obtained with the parameters, $\alpha=$ 2000 and $\tau=0$, tolerance level set as $1 \times 10^{-6}$ as stated in [11]. The decomposed mode with the highest kurtosis value is considered as an effective component for further processing. 


\section{B. Group-Sparse Total Variation (GSTV) denoising}

1) Notation: An $N$-point signal $\mathbf{x} \in \mathbb{R}^{N}, 0 \leq n \leq N-1$ is defined as the column vector,

$$
\mathbf{x}=[x(0), \ldots, x(N-1)]^{T}
$$

The first-order difference matrix is represented by $\mathbf{D}$, i.e.,

$$
\mathbf{D}=\left[\begin{array}{rrrrrr}
-1 & 1 & & & & \\
& -1 & 1 & & & \\
& & \ddots & \ddots & & \\
& & & -1 & 1 & \\
& & & & -1 & 1
\end{array}\right]
$$

The first-order difference of an N-point signal $\mathbf{x}$ is given by Dx where $\mathbf{D}$ is a matrix of $(N-1) \times N$ size.

A vector $\mathbf{v}$ of K-point adjacent samples with starting index $n$ is denoted as:

$$
\mathbf{v}_{n, K}=[v(n), \ldots, v(n+K-1)] \in \mathbb{C}^{K}
$$

2) Model: The noisy data $\mathbf{y} \in \mathbb{R}^{N}$ is described as,

$$
\mathbf{y}_{n}=\mathbf{x}_{n}+\mathbf{w}_{n}
$$

$\mathbf{x} \in \mathbb{R}^{N}$ and $\mathbf{w} \in \mathbb{R}^{N}$ are the clean signal and the noise respectively. The clean signal $\mathrm{x}$ can be estimated by solving the optimization problem:

$$
\arg \min _{\mathbf{x} \in \mathbb{R}^{N}}\left\{F(\mathbf{x})=\frac{1}{2}\|\mathbf{y}-\mathbf{x}\|_{2}^{2}+\lambda \phi(\mathbf{D x})\right\}
$$

where $\phi$ is a penalty function that promotes group sparsity, $\mathbf{v}=\mathbf{D} \mathbf{x} \in \mathbb{R}^{(N-1)}$ as first-order difference of $\mathbf{x}$ which has group sparse behavior and $\lambda$ is the regularization parameter.

The penalty function described in [16] is used in this work,

$$
\phi(\mathbf{v})=\sum_{n}\left[\sum_{k=0}^{K-1}|v(n+k)|^{2}\right]^{1 / 2}
$$

$\mathrm{K}$ denotes the group size. If $\mathrm{K}=1, \phi(\mathbf{v})=\|\mathbf{v}\|_{1}$ and then Equation (9) is standard 1D total variation denoising problem. In this work, $\mathrm{K}$ is set as 3 , then the function $\phi(\mathbf{v})$ is a convex measure of group sparsity. As stated in [13], a positive value of $\lambda$ is selected by trial and error method and in the range of 0.01 to 1 . GSTV uses the computationally efficient and fast converging MM algorithm to minimize $F(x)$ [16].

\section{Results And Discussions}

In order to assess the performance of denoising algorithms, the following parameters were computed and analyzed. (i) RMSE: The root mean square error (RMSE) used to measure the error between denoised signal and simulated PD signal, lower values of RMSE is better denoising algorithm. (ii) CC: Correlation coefficient parameter indicates the highest shape similarity for $c c=1$, on the other hand $c c=-1$ means total asymmetry between the signals. (iii) PAR (in \%): Peak amplitude reduction is calculated using the peak amplitude of the input signal and the peak amplitude of the denoised signal. Lower values of PAR is better denoising algorithm.

The simulated PD signals were subjected to various noise models as discussed in the Section II. A sample PD signal $S_{2}$ which has about twenty pulses at regular time interval is shown in Figure 1(i) along with the noisy PD signal which
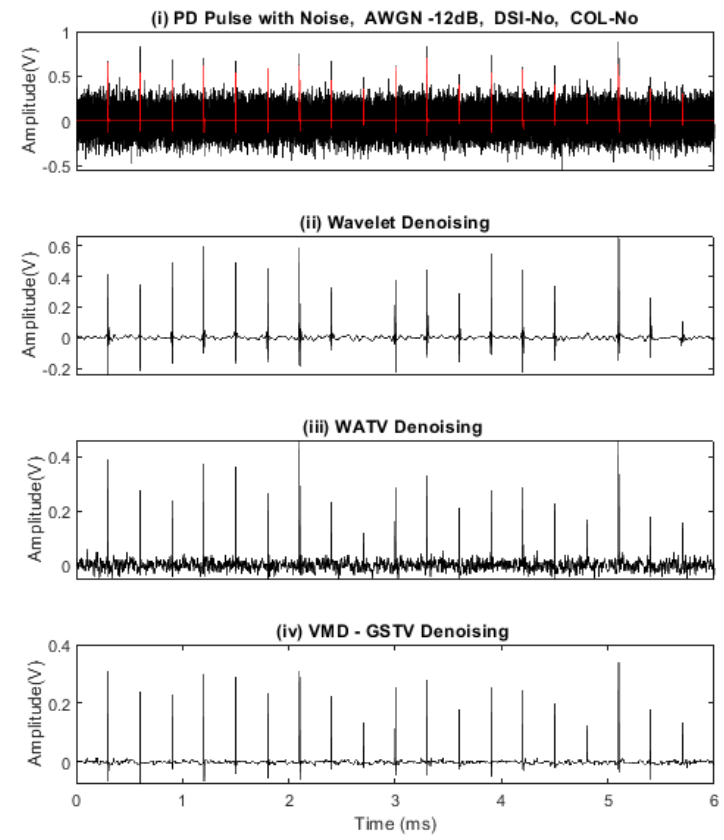

Fig. 1: Illustration of PD signal denoising. (i) PD signal $S_{2}$ (in Red) in presence of additive Gaussian white noise with $\mathrm{SNR}=-12 \mathrm{~dB}$ (in black) and absence of DSI and Color noise; (ii) Denoised signal using wavelet denoising; (iii) Denoised signal using WATV denoising; (iv) Denoised signal using proposed VMD-GSTV denoising.

is corrupted by additive white Gaussian noise with SNR of $-12 d B$ and the denoised signals using Wavelet, WATV and VMD-GSTV denoising methods are shown in Figure 1(ii)-(iv).

The simulated PD signals were processed by three denoising algorithms for ten iterations and the average value of performance indices such as RMSE, CC and PAR are presented in Table II. Though, the VMD-GSTV method is removing the Gaussian noise better than WATV and wavelet methods, it is noted that there is reduction of the pulse amplitude of VMD-GSTV output. It is also observed that few PD pulses are missing in the output of wavelet denoising method during excessive white noise level.

\begin{tabular}{|c|c|c|c|c|}
\hline $\begin{array}{c}\text { Signal with } \\
\text { Noise }\end{array}$ & $\begin{array}{l}\text { Denoising } \\
\text { Methods }\end{array}$ & RMSE & $\mathrm{CC}$ & $\operatorname{PAR}(\%)$ \\
\hline \multirow{3}{*}{ AWGN } & VMD-GSTV & 0.0135 & 0.93 & 46.7 \\
\hline & $W A T V^{1}$ & 0.0177 & 0.73 & 35.4 \\
\hline & Wavelet $^{2}$ & 0.0157 & 0.80 & 9.1 \\
\hline \multirow{3}{*}{$\begin{array}{c}\text { AWGN+ } \\
\text { DSI }\end{array}$} & VMD-GSTV & 0.0181 & 0.90 & 57.3 \\
\hline & $W A T V^{1}$ & 0.0142 & 0.83 & 17.2 \\
\hline & Wavelet $^{2}$ & 0.0174 & 0.78 & 9.1 \\
\hline \multirow{3}{*}{$\begin{array}{c}\text { AWGN+ } \\
\text { DSI+ } \\
\text { Color }\end{array}$} & VMD-GSTV & 0.0192 & 0.77 & 44.1 \\
\hline & $W A T V^{1}$ & 0.0271 & 0.63 & 18 \\
\hline & Wavelet $^{2}$ & 0.0250 & 0.60 & 14.7 \\
\hline
\end{tabular}

Figure 2(a) shows the RMSE value of a PD signal corrupted with various SNR level of white noise. In this condition, VMD-GSTV performs better than the wavelet denoising, and Figure 2(c) shows VMD-GSTV performs better in removing

TABLE II: Performance Comparison of Three Denoising Algorithm

Best performance indicators are in bold font.

1 - WATV proposed in [18] is used as applied for PD in $[19,20]$.

2 - The MATLAB ${ }^{\circledR}$ function 'wden' is used as discussed in [3]. 


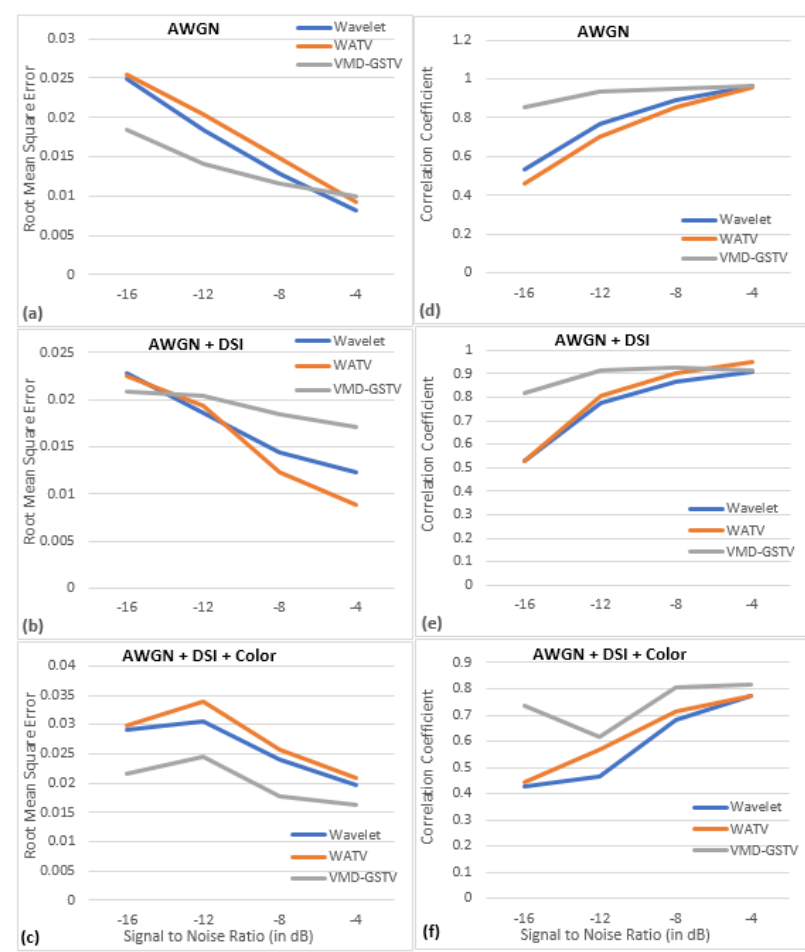

Fig. 2: Mean value of the RMSE and the correlation coefficient of denoised signal for each method: $(\mathrm{a}, \mathrm{d})$ for the white noise only; $(\mathrm{b}, \mathrm{e})$ for the white noise and discrete spectral interference; $(c, f)$ for the white noise, discrete spectral interference and color noise.

white noise, discrete spectral interference and color noise. On the other hand, according to Table II, VMD-GSTV performs low when it is subjected to white and DSI only, however, the $\mathrm{CC}$ is highest as compared to other methods. The PAR value of a specific signal corrupted with three noise models are listed in Table II, wavelet denoising stands out with very low PAR values as compared to other methods. The output amplitude can be normalized to a scale for better representation as presented the results in [8].

Correlation coefficient obtained between simulated PD signal and denoised signal were plotted in Figure 2(d) - 2(f), which clearly indicates that VMD-GSTV performs better than other techniques. In spite of the promising results, the following can be considered in future work:

- Parameters such as penalty function, group size and regularization for GSTV is currently chosen based on iterative method, which can be automated / optimized

- Improvised VMD method to avoid mode-mixing issue

- Selection rule for BLIMF(s) to pick the best mode with that of the PD signal

\section{CONCLUSION}

In this paper, a new method based on VMD-GSTV is proposed for denoising PD signals and the results are compared with wavelet and WATV denoising methods. A synthetic PD signal with white noise, DSI and color noise were considered in this study. The RMSE and CC values are in favour of VMDGSTV based on the results obtained, and peak amplitude reduction of denoised signal is observed. With the comparative study on three denoising methods, the proposed VMD-GSTV method achieved better results in terms of $\mathrm{CC}$ metric and can be potentially extended further.

\section{REFERENCES}

[1] G. C. Stone, "Partial discharge diagnostics and electrical equipment insulation condition assessment," IEEE Transactions on Dielectrics and Electrical Insulation, vol. 12, no. 5, pp. 891-903, 2005.

[2] X. Ma, C. Zhou, and I. Kemp, "Interpretation of wavelet analysis and its application in partial discharge detection," IEEE Transactions on Dielectrics and Electrical Insulation, vol. 9, no. 3, pp. 446-457, 2002.

[3] S. Sriram, S. Nitin, K. Prabhu, and M. Bastiaans, "Signal denoising techniques for partial discharge measurements," IEEE Transactions on Dielectrics and Electrical Insulation, vol. 12, no. 6, pp. 1182-1191, 2005.

[4] X. Zhou, C. Zhou, and I. J. Kemp, "An improved methodology for application of wavelet transform to partial discharge measurement denoising," IEEE Transactions on Dielectrics and Electrical Insulation, vol. 12 , no. 3 , pp. 586-594, 2005.

[5] M. Ghorat, G. B. Gharehpetian, H. Latifi, and M. A. Hejazi, "A New Partial Discharge Signal Denoising Algorithm Based on Adaptive DualTree Complex Wavelet Transform," IEEE Transactions on Instrumentation and Measurement, vol. 67, no. 10, pp. 2262-2272, 2018.

[6] A. Hill, B. G. Stewart, S. G. McMeekin, and G. Morison, "Partial Discharge Signal Denoising Using the Empirical Mode Decomposition,' International Journal of Signal Processing Systems, vol. 1, no. 2, pp. 268-272, 2013.

[7] T. Jin, Q. Li, and M. A. Mohamed, "A Novel Adaptive EEMD Method for Switchgear Partial Discharge Signal Denoising," IEEE Access, vol. 7, pp. 58 139-58 147, 2019.

[8] J. Long, X. Wang, D. Dai, M. Tian, G. Zhu, and J. Zhang, "Denoising of UHF PD signals based on optimised VMD and wavelet transform," IET Science, Measurement \& Technology, vol. 11, no. 6, pp. 753-760, 2017.

[9] Z. Feng, D. Zhang, and M. J. Zuo, “Adaptive Mode Decomposition Methods and Their Applications in Signal Analysis for Machinery Fault Diagnosis: A Review with Examples," IEEE Access, vol. 5, pp. $24301-$ $24331,2017$.

[10] J. Zhang, J. He, J. Long, M. Yao, and W. Zhou, "A new denoising method for UHF PD signals using adaptive VMD and SSA-based shrinkage method," Sensors (Switzerland), vol. 19, no. 7, 2019.

[11] K. Dragomiretskiy and D. Zosso, "Variational mode decomposition," IEEE Transactions on Signal Processing, vol. 62, no. 3, pp. 531-544, 2014.

[12] L. I. Rudin, S. Osher, and E. Fatemi, "Nonlinear total variation based noise removal algorithms," Physica D: Nonlinear Phenomena, vol. 60, no. 1-4, pp. 259-268, 1992.

[13] I. W. Selesnick and I. Bayram, "Total Variation Filtering," Connexions, 2009.

[14] L. Condat, "A Direct Algorithm for 1-D Total Variation Denoising," IEEE Signal Processing Letters, vol. 20, no. 11, pp. 1054-1057, 2013.

[15] S. Zhang, Y. Wang, S. He, and Z. Jiang, "Bearing fault diagnosis based on variational mode decomposition and total variation denoising," Measurement Science and Technology, vol. 27, no. 7, p. 75101, 2016.

[16] I. W. Selesnick and P. Chen, "Total variation denoising with overlapping group sparsity," in 2013 IEEE International Conference on Acoustics, Speech and Signal Processing, no. 4. IEEE, 2013, pp. 5696-5700.

[17] F. G. Vecino, A. R. Linares, F. A. Gomez, C. A. V. Romero, and E. A. del Alamo, "Development of a programmable partial discharge generator for the evaluation of partial discharge measuring devices," in 2018 IEEE 2nd International Conference on Dielectrics (ICD). IEEE, 2018, pp. $1-4$.

[18] Y. Ding and I. W. Selesnick, "Artifact-Free Wavelet Denoising: Nonconvex Sparse Regularization, Convex Optimization," IEEE Signal Processing Letters, vol. 22, no. 9, pp. 1364-1368, 2015.

[19] I. Mitiche, G. Morison, A. Nesbitt, M. H. Narborough, P. Boreham, and B. G. Stewart, "An evaluation of total variation signal denoising methods for partial discharge signals," 2017 INSUCON - 13th International Electrical Insulation Conference (INSUCON), pp. 1-5, 2017.

[20] P. V. d. C. Batista, H. d. O. Mota, G. M. Ferreira, F. T. d. A. Silva, and F. H. Vasconcelos, "Wavelet Total Variation Method Applied to Partial Discharge Signal Denoising," in 2018 3rd International Symposium on Instrumentation Systems, Circuits and Transducers (INSCIT). IEEE, 2018, pp. 1-6. 